\title{
STUDY OF THE STELLAR POPULATIONS IN NGC 3516
}

\author{
M. SEROTE-ROOS \\ DAEC, Observatoire de Meudon, 92195 Meudon, France \\ Centro Astrofisica, Universidade Porto, 4100 Porto, Portugal \\ AND \\ C. BOISSON AND M. JOLY \\ DAEC, Observatoire de Meudon, 92195 Meudon, France
}

Our aim is to quantify the stellar population within the nuclear regions of AGN by means of long slit spectroscopic observations in the optical range (5000-10000 $\AA$ ), including MgI, NaI, TiO, CN, CaIIT. We wish to determine whether there is a relationship between central activity and stellar population, and to determine the nature of the featureless continuum.

For the well-known type 1 Seyfert galaxy, NGC 3516, our results show that the equivalent width of the lines, including CaIIT, are decreasing towards the centre. It can be interpreted as a dilution of the nuclear stellar component by an additional spectral component if we postulate that the stellar population is identical in the nucleus and in the surrounding region.

Synthetic models of this stellar population have been obtained. The total flux at $5450 \AA$ of the extranuclear region is dominated $(65 \%)$ by the emission of cool stars (mainly $\mathrm{K}$ ) from all luminosity classes (V, III, I) including a high contribution of SMR stars (30\%). The contribution of blue stars (35\%) is restricted to $\mathrm{A}$ and $\mathrm{F}$ types. An internal reddening of $E_{B-V}=0.07$ is assumed.

We have subtracted the stellar contribution to the nuclear spectrum. All absorption features disappear: we are left with a featureless continuum. The most obvious explanation is the well-known non-thermal power-law continuum observed in AGN. However, if the featureless continuum is not of non-thermal origin, it should arise from an additional stellar population characterised by hot featureless stars, i.e. O and B stars. So, a stellar population gradient may still exist in the AGN, hidden by the emission line spectrum. However, the spectrum of the possible OB stars has to be strongly reddenned to agree with the featureless continuum. 